\title{
La influencia altiplánica incaica en los textiles del Período Tardío en Arica ${ }^{1}$
}

Gabriela Carmona $S^{2}$

\section{RESUMEN}

Se presenta una investigación que intenta caracterizar los textiles de afiliación inca encontrados en sitios arqueológicos tardíos de Arica, norte de Chile. Se analizan los patrones estructurales y decorativos presentes en los textiles tardíos, entregando evidencias de multietnicidad y evaluando la capacidad de estos materiales para representar identidades étnicas, de género u otras.

\begin{abstract}
This research presents an investigation about the characterization of the inca's textiles found in archaeological late sites from Arica, northern Chile. This paper analyzes structure and decorative patterns present in the late textiles, showing multiethnicity evidences, and evaluating the textile capacity to represent ethnic, social and gender identity.
\end{abstract}

\section{Introducción}

Este trabajo tiene el propósito de presentar parte de la investigación sobre la caracterización de las

1 Este trabajo forma parte de la Memoria para optar al Título de Arqueóloga, investigación inserta dentro del proyecto FONDECYT 1970840 "Etnicidad e identidad cultural: Una visión desde la textilería prehispánica" a cargo de Liliana Ulloa y las coinvestigadoras Jacqueline Correa. Vivian Gavilán y Vivien Standen.

2 Museo Fonck, Viña del Mar, Casilla 951. Email: MuseoFonck@123click.cl prendas textiles incas presentes en los sicios arqueológicos tardíos del extremo norte de Chile, cuyo objetivo es definir los patrones estructurales y decorativos que están presentes en los materiales textiles del Período Tardío en el extremo norte de Chile, evaluando las evidencias sobre la multietnicidad del territorio, la permanencia y la permeabilidad de los materiales textiles y su capacidad para representar identidades étnicas, sociales, de género u otras.

Se trata de establecer cuáles fueron los cambios identificados en los textiles pertenecientes a las poblaciones que ocuparon los oasis del sector del valle de Camarones, el valle de Alto Ramírez y la costa de Arica, en el Período Tardío, en relación al Período de Desarrollos Locales o Período Intermedio Tardío, a través del análisis y comparaciones de los tejidos encontrados en los cementerios de Camarones 9, Azapa 15 y Playa Miller 3. Una vez señalados los cambios y sus variables podemos evaluar distintas interpretaciones sobre las causas y consecuencias de éstos, y si se vinculan a diferencias étnicas, de status, sociales, de género u otras.

\section{Los tejidos incas en Arica}

Cuando nos referimos a los tejidos de este período, nos encontramos con patrones nuevos, especialmente en el aspecto decorativo que van a estandarizar y uniformar la producción textil, imponiéndose ante una gran variedad y riqueza de diseños desarrollados por las poblaciones locales en el Período Intermedio Tardío. Asociado a esta nueva situación, se produce un cambio en la manera cómo se utilizan y distribuyen los textiles. En "La 
Organización Económica del Estado Inca," (Murra 1978:107), señala que los tejidos

"servian en diferentes momentos y ocasiones como simbolo de elevada posición social o como señal de una ciudadanía forzosa..."

Sin embargo, hasta ahora se desconoce qué vestían los habitantes de las colonias o comunidades ocupadas o conquistadas, qué ocurre con los tejidos tradicionales de los grupos étnicos bajo su dominio y dónde se producen y distribuyen los tejidos.

Creemos que las prendas textiles cumplen un rol significativo como elementos diferenciadores, por lo que al abordar el estudio de una colección de tejidos "incas" o asociados a la esfera de dominio del Tawantinsuyu, a través del análisis de los materiales textiles de los sitios Camarones 9, Azapa 15 y Playa Miller 3, éstas y otras interrogantes podrán contar con nuevos elementos que tiendan a su explicación.

Sabemos que el imperio incaico tuvo como política no imponerse de manera absoluta en sus colonias, privilegiando la diversidad de costumbres. Así cada ayllu o parcialidad conservaba sus rasgos tradicionales en la manera de vestirse, que también correspondían a distintas exigencias ambientales. Estas concesiones de mantener los patrones de vestimenta de cada región anexada, constituía una ordenanza inca porque facilitaba la identificación de los individuos. No obstante, la condición de Arica no era el de una colonia, ya que estaba considerada dentro del territorio de expansión del Collasuyo, por lo tanto constituye una área de difícil adscripción si tomamos en cuenta que la influencia incaica estuvo siempre permeada por los grupos altiplánicos contemporáneos.

Es muy difícil el estudio de los patrones estructurales y estilísticos de los tejidos asociados a contextos tardíos con influencia inca, porque el registro arqueológico en muchos de los sitios tardíos no ha sido riguroso, restándole importancia a los materiales textiles. Es así que la naturaleza de los materiales es dicotómica: por una parte nos encontramos con textiles más bien considerados "marginales" porque presentan rasgos locales con reparaciones que aluden a querer asimilarse a lo inca y por otro lado, en forma aislada tejidos cumbi que evidencian la presencia de representantes inca, o pueden ser considerados como producto del intercambio y distribución de bienes por parte del Estado inca. Lo cierto es que la mayoría de los materiales textiles expresan una influencia indirecta que refuerza la idea que las poblaciones altiplánicas hacían las veces de intermediarios en la introducción de patrones incas.

En relación al valle de Azapa y costa de Arica, recientemente se ha logrado definir diferentes estilos textiles, caracterizándose a los estilos locales y a los foráneos (Agüero y Horta 1997 Ms). Estos estilos textiles además se corresponden en forma coherente con los tipos cerámicos representativos de diferentes grupos asentados en el área.

Tomando la información de Agüero y Horta (1997 Ms), basada en el análisis de los materiales textiles correspondientes a bolsas agrícolas, bolsas chuspas e inkuñas de los sitios PLM-3 y PLM-9 de la costa y AZ-8 del valle, en el Período Intermedio Tardío, vemos que las bolsas chuspas dan cuenta de la existencia de al menos seis estilos: Estilo San Miguel (Grupo 1A); Estilo San MiguelPocoma (Grupos 1 A y 3); Estilo Pocoma (Grupos 1D y 1G); Estilo Costero (Grupo 1B y 1F); Estilo Tarapaqueño (Grupo H), y Estilo Incanizado (Grupo 1I). Este último correspondería a las primeras evidencias de influencia incaica en la textilería de Arica y la primera caracterización de una de sus manifestaciones.

En el caso de las inkuñas se han definido cuatro estilos: San Miguel (Grupo 2B); San MiguelPocoma (Grupo 2D); Pocoma (Grupo 2A), y Costero (Grupos 1H, 2C, 2E, 2F y 2G).

Estas investigadoras señalan que las principales variaciones que se producen en la textilería de Arica durante el Período Intermedio Tardío, se expresan a través de la apariencia de las prendas.

Evidencias directas de la influencia inca en los materiales textiles se registran en los componentes más tardíos de los sitios PLM-3 y PLM-4, donde se encuentran prendas cumbi, ejemplares de la textilería inca imperial.

Refiriéndose a las diferencias y semejanzas 
estilísticas e iconográficas registradas entre los materiales de la costa y el valle, afirman que en la primera mitad del Período Intermedio Tardío existe mayor homogeneidad entre las dos áreas, interpretada como resultado de una unidad cultural. En la segunda mitad del período, se aprecia una proliferación de estilos post San Miguel en la costa, de características desconocidas en la textilería local, lo que podría indicar la presencia de grupos originarios de otros lugares (costa sur del Perú, costa de Tarapacá). Por tanto, la evidencia textil de fines del Intermedio Tardío respaldaría la información etnohistórica de documentos del siglo
XVI, acerca de la coexistencia en la costa de Arica de pescadores de Tarapacá, Tacna e Ilo (Hidalgo y Focacci 1986).

De acuerdo a lo señalado en algunos estudios en relación a los tejidos del Período Intermedio Tardío se esbozan cambios al contrastarlos con los tejidos encontrados en contextos más tardíos o con influencia inca, tanto en su forma, como en su decoración, los que resumimos a continuación. Se utilizan preferentemente colores naturales para el color de fondo de cualquier tipo de textil, reservando los colores teñidos (de gama limitada) para

Cuadro 1. Diferencias formales entre los tejidos de los períodos Intermedio Tardío y Tardío

\begin{tabular}{|c|c|c|}
\hline FORMA TEXTIL & PERIODO INTERMEDIO TARDIO & PERIODO TARDIO \\
\hline$I N K U \tilde{N A}$ & - Abundancia de inkuñas. & - Desaparecen gradualmente. \\
\hline BOLSA FAJA & $\begin{array}{l}\text { - Presencia de bolsas fajas profusamente } \\
\text { decoradas. }\end{array}$ & $\begin{array}{l}\text { - Las bolsas fajas se vuelven más toscas y } \\
\text { gradualmente desaparecen (Ulloa 1982: } \\
\text { 102). }\end{array}$ \\
\hline TALEGA & - Su cantidad no es numerosa. & $\begin{array}{l}\text { - Se hacen más frecuentes las talegas } \\
\text { (Ulloa 1982: 105). }\end{array}$ \\
\hline BOLSA CHUSPA & $\begin{array}{l}\text { - Las bolsas chuspas generalmente sólo } \\
\text { contienen hojas de coca (Agiiero y Horta } \\
1997 \mathrm{Ms} \text { ). } \\
\text { - El número de refuerzos y reparaciones } \\
\text { de las piezas es escaso. } \\
\text { - Las asas son acordes con el diseño } \\
\text { general de la pieza, en muchos casos sólo } \\
\text { son trenzados o cuerdas y en otros no se } \\
\text { presentan. }\end{array}$ & $\begin{array}{l}\text { - El contenido de las bolsas chuspas varía } \\
\text { y se amplia desde hojas de coca, a } \\
\text { plumas, pelos, palomitas de maíz y otros, } \\
\text { en el Tardío (Agüero y Horta } 1997 \mathrm{Ms} \text { ). } \\
\text { - Se aprecian mayores refuerzos en los } \\
\text { bordes y orillas con festón anillado } \\
\text { simple. } \\
\text { - Las asas son más gruesas, tejidas a telar } \\
\text { y presentan diseños geométricos en } \\
\text { franjas horizontales (Ulloa 1982: 105). }\end{array}$ \\
\hline CAMISA & $\begin{array}{l}\text { - Se presentan formas trapezoidales y } \\
\text { semitrapezoidales. }\end{array}$ & $\begin{array}{l}\text { - Cambian las formas de las camisas, las } \\
\text { cuales en general son cuadradas o } \\
\text { rectangulares. }\end{array}$ \\
\hline GORRO & $\begin{array}{l}\text { - Las formas más documentadas para este } \\
\text { período son: Casco de forma cupular, } \\
\text { Gorro de fibra vegetal tipo "birrete" } \\
\text { circular, Gorro tipo "pasamontañas", en } \\
\text { algunos casos con aplicaciones de plumas } \\
\text { (Berenguer 1993). }\end{array}$ & $\begin{array}{l}\text { - Cambian las formas de los gorros. El } \\
\text { tipo más usado presenta la forma de fez o } \\
\text { cono truncado con diseños geométricos } \\
\text { de triángulos y ganchos contrapuestos en } \\
\text { diversos colores y que a veces incluyen } \\
\text { un penacho de plumas y placas de metal } \\
\text { como adornos accesorios (Berenguer } \\
\text { 1993). }\end{array}$ \\
\hline SAQUITO & - No se registra la presencia de saquitos. & - Surgen las formas de "saquitos". \\
\hline
\end{tabular}




\section{FORMA TEXTIL}

\section{PERÍODO INTERMEDIO TARDIO}

-Se amplía el bagaje de motivos decorativos, son comunes los seres antropo y zoomorfos.
BOLSAS FAJA
INKUNA
BOLSAS CHUSPA
BOLSA CHUSPA

CAMISA
-En un primer momento, en la fase San Miguel Temprano, predominan con fuerza los motivos figurativos antropomorfos y zoomorfos. Más tarde, en la fase San Miguel Tardío, son comunes las composiciones geométricas. Luego, en la fase San Miguel - Pocoma se presentan superficies totalmente decoradas, volviendo a predominar motivos zoomorfos complementados por composiciones geométricas (Horta 1999). - Se utiliza una amplia variedad de
colores, aumentando su gama.

- Presentan listados verticales polícromos de variada ubicación al interior de la pieza (Horta 1999: 81).
- Disminuye la variedad de motivos decorativos utilizados, desaparecen motivos y se introducen otros, entre los cuales se destacan los motivos "Hexágonos concéntricos", "Rombos concéntricos". "Rombos centrales con silueta de rombos escalerados", "Rombos con punto central en sucesión vertical" y "Reloj de arena." Se privilegia la ornamentación con listados (Ulloa 1982: 102).

- Se reduce la variedad y cantidad de técnicas utilizadas para lograr los motivos decorativos. Desaparece la técnica textil de doble faz (Ulloa 1982: 102).

- Disminuyen los colores teñidos especialmente los verdes y azules, sólo se mantienen los tonos rojos y anaranjados. Se usan preferentemente los colores naturales de la lana de camélidos (Ulloa 1982: 105).

- Disminuye la cantidad de motivos, se vuelven comunes los motivos geométricos simples: letra $Z$ en sucesión vertical, ganchos en miniatura, ganchos continuos, voluta "s" individual y con prolongaciones, voluta "s" de cabezas entrelazadas, voluta "s" de cabezas entrelazadas en imagen-espejo, rombos radiados de seis ejes. Los motivos chevrón y su variante invertida ("espiga") son elementos recurrentes en los tejidos incaicos. $\mathrm{Al}$ igual que el rombo con punto central en múltiples variantes (a) individual, (b) en sucesión vertical, (c) como segmento en módulos de rombos adyacentes, y (d) seccionado longitudinalmente (Agüero y Horta 1997 Ms).

- Se utilizan preferentemente colores naturales, reservando los colores teñidos (de gama limitada) para las franjas decoradas (Aguiero y Horta 1997 Ms).

- En general son listadas o lisas en colores naturales (Ulloa 1982: 102). 
las franjas decoradas. Este rasgo es identificado en el grupo de chuspas (1I) en la clasificación de Agüero y Horta (1997 Ms).

En el caso de las camisas, aquellas típicamente incaicas estaban tejidas en tapicería enlazada con un esquema propio de composición de patrón muy geométrico, utilizando preferentemente motivos de damero y líneas verticales. Este tipo de camisas no se ha registrado fuera del área nuclear del territorio inca, por lo que se cree era destinado sólo a la elite del estado.

\section{Conclusiones preliminares}

Estos cambios, adaptaciones o transformaciones, tanto de las formas predominantes como de los elementos del diseño nos revelan diferencias culturales. Lamentablemente los contextos donde es posible recuperar este tipo de información son escasos, por tanto la mayoría de los cambios no han sido comprobados estadísticamente, sin embargo han sido observados por investigadores a lo largo de varios años de estudio.

En general, a través de estos indicadores se tiende a confirmar que existían distintas normas de composición y la presencia de motivos particulares que identificaban a diversos lugares y etnias dentro del imperio. Por ejemplo, se observa que los miembros del Collasuyu (parte sur, que incluye Chile) usaban en gran medida el listado vertical en sus camisas, y además existen preferencias por aquellas técnicas que permiten esa imagen, como lo son las técnicas de faz de urdimbre.

En los tejidos inca imperiales, una característica importante de las camisas es la incorporación de los tocapus, (cuadrados de aproximadamente 10 x $10 \mathrm{~cm}$ dispuestos en bandas horizontales o cubriendo la superficie total de la prenda que presentan en su interior una serie de motivos geométricos que podrían interpretarse como ideogramas), lo que nos demuestra las grandes diferencias posibles de encontrar entre los tejidos incas y los que se popularizaron en el Período Tardío en Arica.

Los principales rasgos que permanecen sin variaciones son las técnicas estructurales de elaboración de los textiles. Notamos que las formas texti- les asociadas a labores domésticas, como los sacos, bolsas agrícolas o talegas y wayuñas no presentan grandes cambios en su decoración a lo largo de, aproximadamente cinco siglos, ya que algunas se siguen tejiendo hoy en día, en comunidades aymaras, con los mismos diseños que en épocas preincaicas. En cambio las formas que están vinculadas a ceremonias y celebraciones especiales, como las bolsas chuspas, las inkuñas, las bolsas fajas y las camisas más finas, cambian profundamente en su estilo decorativo o simplemente desaparecen a raíz de la influencia incaica que gradualmente impone sus cánones de diseño y ornamentación.

Resumiendo, vemos que el Período Tardío en Arica se caracteriza por una disminución en la producción textil local, que va acompañada de un empobrecimiento de expresión decorativa y pérdida de identidad en sus materiales, los que son reparados y reutilizados, creemos que al influjo del dominio de las poblaciones altiplánicas, las cuales privilegiaban la explotación de los recursos del área para integrar el sistema de distribución e intercambio que imponía la dominación incaica. La manifestación de esta situación la vemos reflejada en la presencia de dos tipos predominantes de evidencia textil:

1.- Tejidos de factura local con reparaciones que imitan la usanza inca, p.e. chuspas con asas con motivos geométricos cosidos.

2.- Tejidos cumbi (escasos y encontrados principalmente en la costa).

El primero de estos tipos o estilos de tejidos podría constituir el tejido altiplánico, el que no se identifica claramente, tal vez porque combina los elementos locales con los adquiridos por sus contactos con los incas.

En el caso de la presencia de tejidos cumbi en la costa, esto podría deberse a testimonios de soberanía por parte de personeros del imperio inca en áreas más lejanas o de importancia estratégica como el litoral.

\section{Agradecimientos}

Por último, agradezco a los organizadores de este 
simposio por invitarme a presentar este trabajo, que sólo pretende rescatar y sistematizar varios años de estudio por parte de numerosos investiga- dores de los textiles arqueológicos del norte grande de Chile, entre los que se destacan: Liliana Ulloa, Carolina Agüero y Helena Horta.

\section{BIBLIOGRAFIA}

AGÜERO, C. y H. HORTA. Los textiles del Período Interme1997 Ms dio Tardío del valle de Azapa y costa de Arica. Informe final Proyecto FONDECYT 1960113.

BERENGUER, J. Gorros, identidad e interacción en el de1993 sierto chileno antes y después del colapso de Tiwanaku. Identidad y prestigio en los Andes. Gorros, turbantes y diademas, pp. 41-64, Museo Chileno de Arte Precolombino, Santiago.

GUAMAN POMA DE AYALA. F. Nueva coronica y buen 1993 [1616] gobierno. F. Pease (Ed.), Fondo de la Cultura Económica, 3 vols. Sección de Obras de Historia, Lima.

HIDALGO, J. y G. FOCACCI. Multietnicidad en Arica. Siglo
1986 XVI, evidencias etnohistóricas y arqueológicas. Chungara 16-17:137-148, Arica

HORTA. H. Estudio iconográfico de textiles arqueo1999 lógicos del valle de Azapa, Arica. Chungara 29/1:81-108, Arica.

MURRA, J.V 1978

La organización del Estado Inca. Instituto de Estudios Peruanos. Colección América nuestra. Siglo XXI Editores, México.

ULLOA, L. Estilos decorativos y formas textiles de 1982 poblaciones agromarítimas, extremo norte de Chile. Chungara 8:109-136, Arica. 


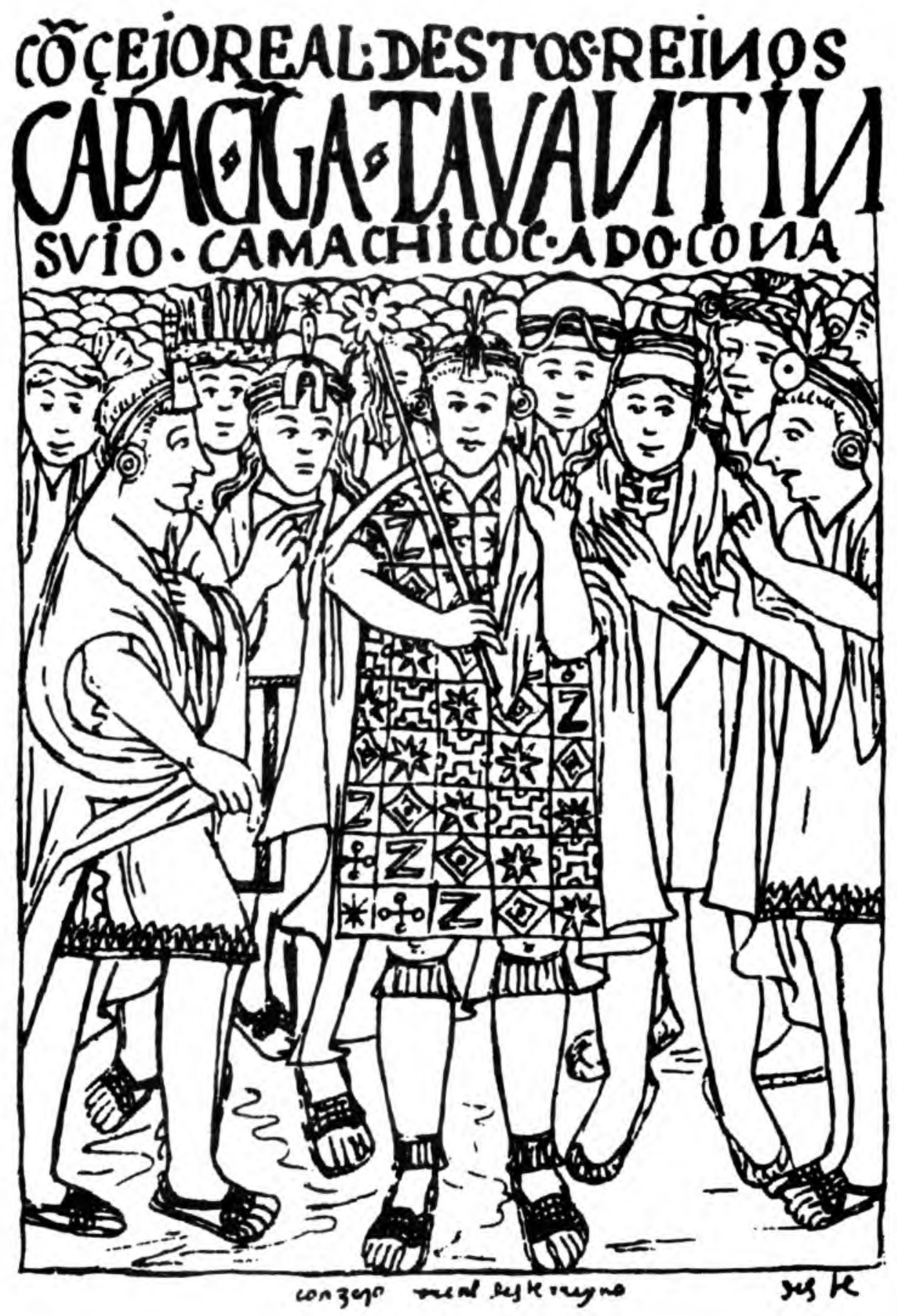

Figura 1. Dibujo que representa los grupos étnicos que conformaban el Tawantinsuyu con sus propias vestimentas (Guamán Poma de Ayala 1993 [1616]) 


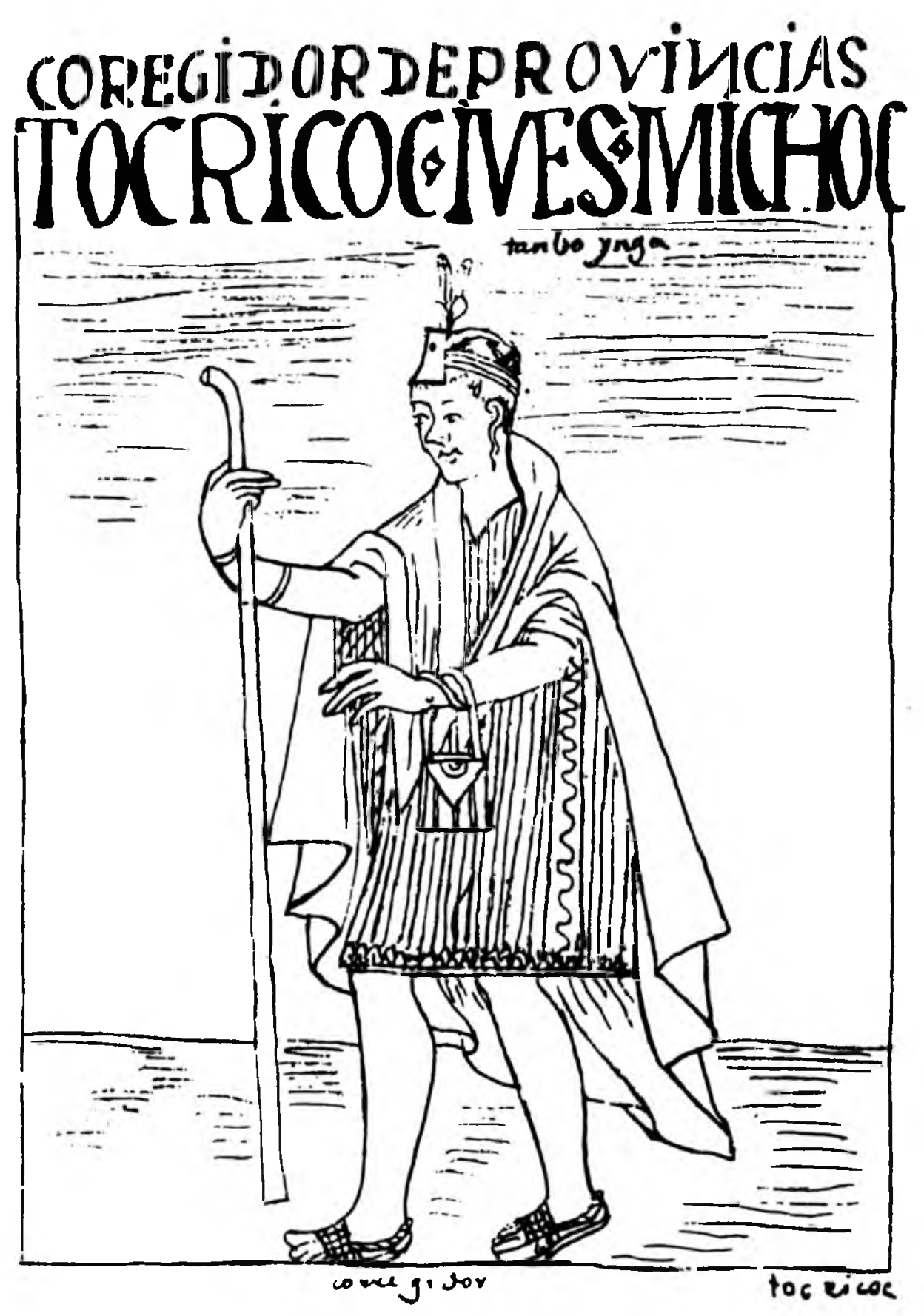

Figura 2. Dibujo que representa a un miembro del Collasuyo (Guamán Poma de Ayala 1993 [1616]) 

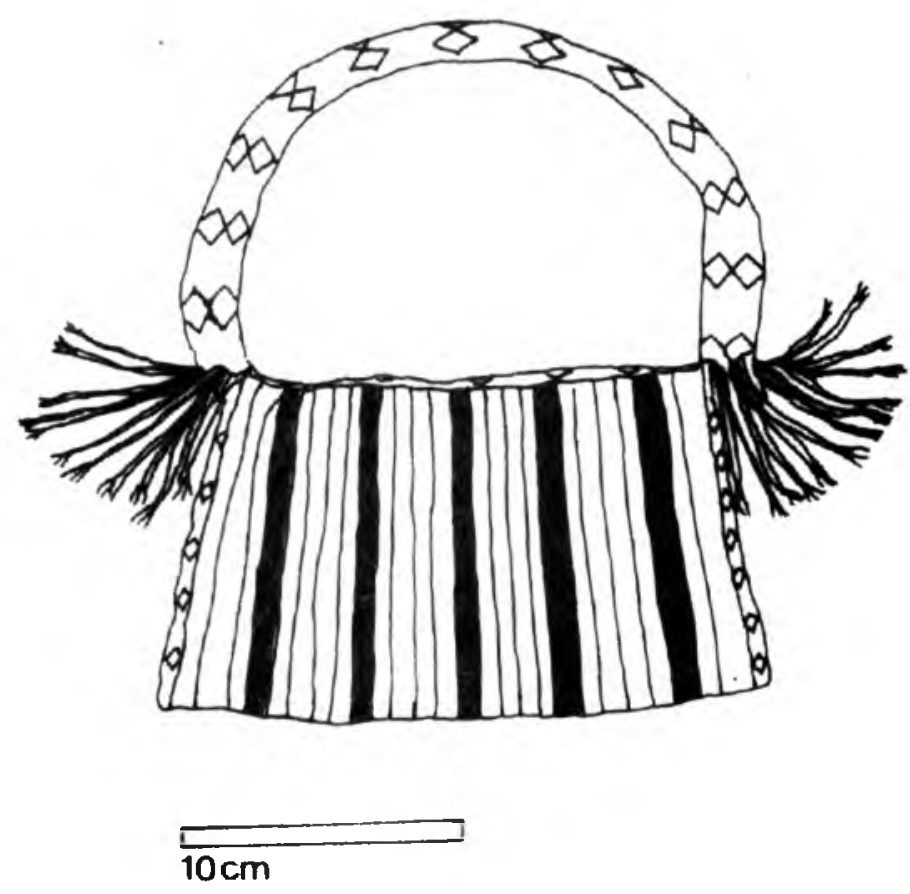

Figura 3. Chuspa inca, sitio PLM-4

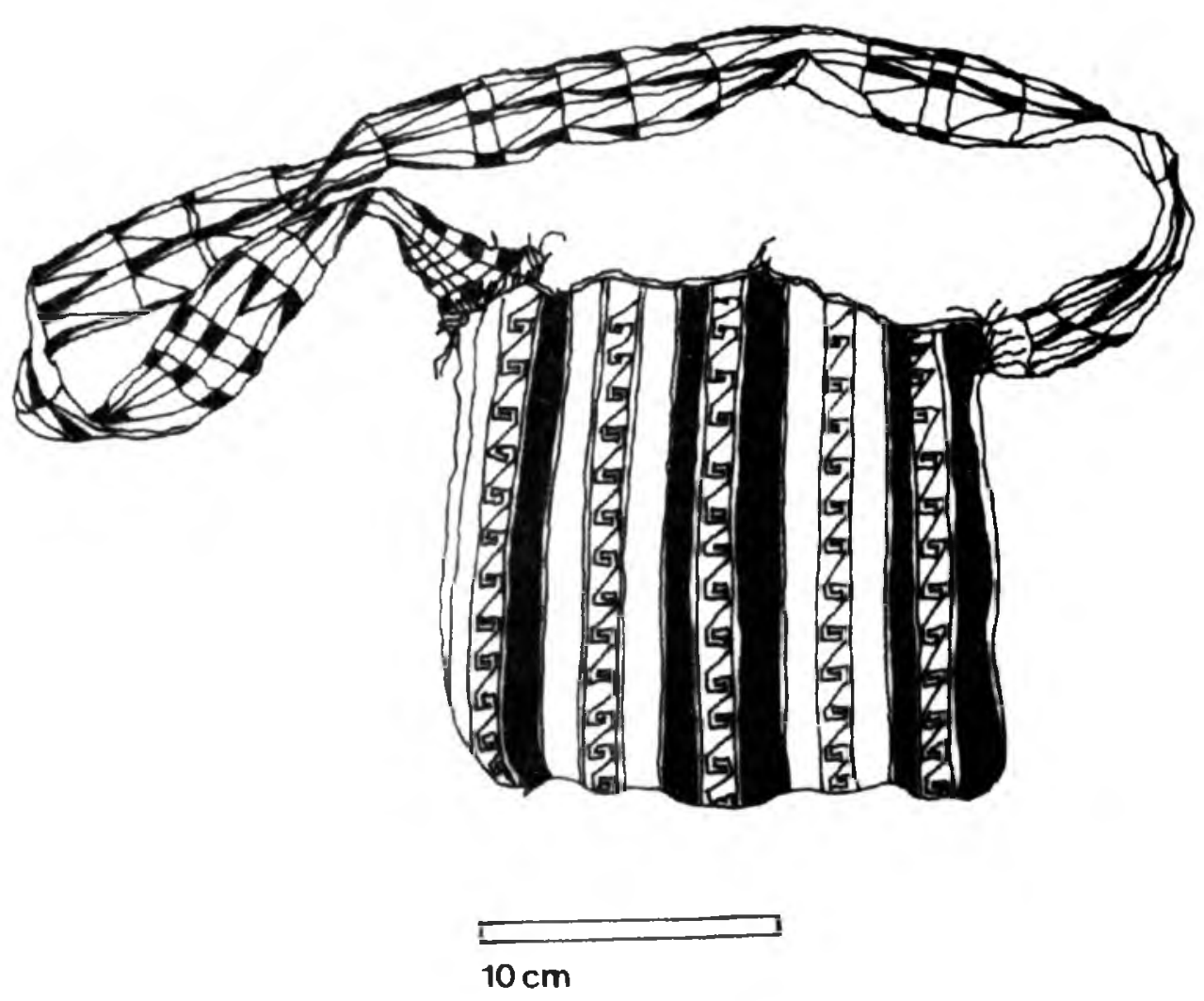

Figura 4. Chuspa ariqueña incanizada, sitio PLM-6 
